\title{
On a PDE Arising in One-Dimensional Stochastic Control Problems
}

\author{
Ricardo Josa-Fombellida • \\ Juan Pablo Rincón-Zapatero
}

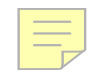

\begin{abstract}
The paper provides a systematic way for finding a partial differential equation that directly characterizes the optimal control, in the framework of onedimensional stochastic control problems of Mayer type, with no constraints on the controls. The results obtained are applied to continuous-time portfolio problems.
\end{abstract}

Keywords Dynamic programming · Stochastic control · Quasilinear parabolic equation · Investment problems

\section{Introduction}

The paper provides a systematic way for finding a partial differential equation (PDE) that can be applied directly to the optimal control in one-dimensional stochastic control problems of Mayer type, where there are no constraints on the controls or, more generally, where the optimal control is interior to the control region. This new PDE is obtained from the optimality conditions of the stochastic maximum principle, and is equivalent to the Hamilton-Jacobi-Bellman (HJB) equation.

\footnotetext{
Communicated by H J Pesch

We wish to thank an Associate Editor and two referees for helpful comments Both authors gratefully acknowledge financial support from the Spanish Ministerio de Ciencia e Innovación under project ECO2008-02358 The first author is also supported by Consejería de Educación de la Junta de Castilla y León (Spain) under project VA056A09

R Josa-Fombellida Departamento de Estadística e Investigación Operativa, Universidad de Valladolid, 47005 Paseo Prado de la Magdalena s/n, Valladolid, Spain

e-mail: ricar@eio uva es

J P Rincón-Zapatero $(\bowtie)$

Departamento de Economía, Universidad Carlos III de Madrid, 28903 C/Madrid 126, Getafe, Madrid, Spain

e-mail: jrincon@eco uc3m es
} 
Though the initial idea of obtaining a system of PDEs for the optimal control appears in [1] in connection with deterministic control problems, the main antecedents of this paper are: [2] and [3] in deterministic differential games; [4], in stochastic control problems, where the diffusion parameter of the state process is independent of the control variables; [5] in the Merton problem; and [6] in a model of optimal liquidation in illiquid markets. In all these papers, the use of the PDE for optimal control has proved to be useful. The objective of this paper is to extend the approach to the one-dimensional stochastic control problem of Mayer type, where there is no running payoff functional, but the diffusion term of the state process depends on the control variable.

Some comments about our assumptions are in order here. We are aware that they are rather strong, as they involve the regularity imposed on the coefficients of the controlled process for the applicability of the stochastic maximum principle, as well as the interiority of the optimal process, which is supposed to be continuously differentiable. These hypotheses are used to find a PDE for the optimal control, and we can think of them as a heuristic method to find new information on the optimal solution, as it is the derivation of the HJB equation for the value function. However, once the PDE is obtained, it can be checked ex-post that the smoothness and interiority assumptions hold and that the solution of the PDE is indeed the optimal solution, even if the requirements of the maximum principle do not hold. This is shown in detail in Merton's problem studied in Sect. 5.2. Thus, the main proposal of the paper is to use the PDE we find as the point of departure to further investigate the optimal solution, as an alternative to the HJB equation.

The paper is organized as follows. In Sect. 2 we present the control problem, as well as some definitions and notations. In Sect. 3 we obtain necessary optimality conditions in the form of PDEs that the adjoint feedback function and the optimal control must satisfy. The relationship between the new PDEs and the HJB equation is shown in Sect. 4, and a sufficient optimality condition is given in terms of a verification theorem in [7]. Section 5 contains applications of the theory to linear models in the dynamics. In particular, the existence of a solution is shown for the Merton problem with deterministic coefficients for a class of utility functions having a bounded relative risk tolerance index. In Sect. 6 models with a multiplicative structure in the dynamics are introduced. It turns out that some simple assumptions on the data allow us to solve a wide range of models of this type, from which we include an extension of Merton's problem to situations, where the investor's decisions may influence the evolution of the stochastic price process of the risky asset. The paper ends with some conclusions in Sect. 7.

\section{The Control Problem}

In this section the framework for the stochastic control problem to be considered is presented. First we shall introduce some useful notations. Throughout this paper, given a differentiable function $h: \mathbb{R}^{n} \rightarrow \mathbb{R}$, we will denote by $h_{y}$ the partial derivative of $h$ with respect to the variable $y$ and, if $n=1$, by $h^{\prime}$ the derivative of $h$ with respect to a variable other than time, and by $\dot{h}$ the derivative with respect to the timevariable $t$. The notation is analogous for the partial derivatives of second order. We 
will denote total derivative by $\partial / \partial x$. We say that a function $h$ is of class $C^{k}$ with respect to $y$ iff the $k$ th partial derivative of $h$ with respect to $y$ is continuous. For a function $h$ that depends on $(t, y)$, we say that the function is of class $C^{1,2}\left(C^{1,1}\right)$ iff both $h_{t}$ and $h_{y y}\left(h_{y}\right)$ are continuous. Vectors $v \in \mathbb{R}^{n}$ are row vectors and $v^{i}$ is the $i$ th component; finally, ${ }^{\top}$ denotes transposition.

Let $[0, T]$ be a time interval with $0<T<\infty$ and let $\left(\Omega, \mathcal{F},\left\{\mathcal{F}_{t}\right\}_{t \in[0, T]}\right.$, P) be a complete filtered probability space. Assume that on this space a $\ell$-dimensional Brownian motion $\{w(t)\}_{t \in[0, T]}$ be defined. Let $\mathrm{E}$ denote expectation under the probability measure $P$.

The state space is $\mathbb{R}$ and $U \subseteq \mathbb{R}$ is the convex control region. A $U$-valued control process $\{u(s)\}$ defined on $[t, T] \times \Omega$ is an $\mathcal{F}_{s}$-progressively measurable map $(r, \omega) \rightarrow$ $u(r, \omega)$ from $[t, s] \times \Omega$ into $U$, that is, $\mathcal{B}_{s} \times \mathcal{F}_{s}$-measurable for each $s \in[t, T]$, where $\mathcal{B}_{s}$ denotes the Borel $\sigma$-field on $[t, s]$. For simplicity, we will denote $u(t)$ as $u(t, \omega)$.

The state process $X \in \mathbb{R}$ obeys the controlled stochastic differential equation (SDE)

$$
d X(s)=f(s, X(s), u(s)) d s+\sigma(s, X(s), u(s)) d w(s), \quad s \geq t,
$$

with initial condition $X(t)=x$. An important feature of the above equation is that the drift, $f$, and the noise coefficient, $\sigma$, both depend on the control variable, $u$. Here, $\sigma$ is a vector with $\ell$ components.

Definition 2.1 (Admissible control) A control $\{u(t)\}_{t \in[0, T]}$ is called admissible iff

(i) for every $(t, x)$, the $\operatorname{SDE}(1)$ with initial condition $X(t)=x$ admits a pathwise unique strong solution;

(ii) there exists some measurable function $\phi:[0, T] \times \mathbb{R} \longrightarrow U$, such that $u$ is in relative feedback to $\phi$, i.e. $u(s)=\phi(s, X(s))$ for every $s \in[0, T]$.

Let $\mathcal{U}(t, x)$ denote the set of admissible controls corresponding to the initial condition $(t, x) \in[0, T] \times \mathbb{R}$.

Given the initial data $(t, x) \in[0, T] \times \mathbb{R}$, the criterion to be maximized is

$$
J(t, x ; u)=e^{-\delta(T-t)} \mathrm{E}_{t x}\{S(T, X(T))\},
$$

in the class of controls $u \in \mathcal{U}(t, x)$, where $\mathrm{E}_{t x}$ denotes conditional expectation with respect to the initial condition $(t, x)$. The constant $\delta \geq 0$ is the discount factor. All the functions $f:[0, T] \times \mathbb{R} \times U \longrightarrow \mathbb{R}, \sigma:[0, T] \times \mathbb{R} \longrightarrow \mathbb{R}^{1 \times \ell}, S:[0, T] \times \mathbb{R} \longrightarrow \mathbb{R}$, are assumed to be continuous. They are also of class $C^{2}$ with respect to $(x, u)$ and of class $C^{1}$ with respect to $t$.

The value function is defined as $V(t, x)=\sup _{u \in \mathcal{U}(t, x)} J(t, x ; u)$. An admissible control $\widehat{u} \in \mathcal{U}$ is optimal iff $V(t, x)=J(t, x ; \widehat{u})$ for every initial condition $(t, x)$.

The classical method for determining feedback solutions in a control problem is based on finding the value function through the HJB equation and the optimal control from that. It is well known that, if $V$ is of class $C^{1,2}$, then it satisfies the HJB 
equation

$$
\begin{aligned}
& V_{t}(t, x)+\max _{u \in U} G\left(t, x, u, V_{x}(t, x), V_{x x}(t, x)\right)=\delta V(t, x), \quad \forall(t, x) \in[0, T) \times \mathbb{R}, \\
& V(T, x)=S(T, x), \quad \forall x \in \mathbb{R},
\end{aligned}
$$

and the maximizing argument is optimal if it is admissible; see [7]. Here

$$
G(t, x, u, p, P)=f(t, x, u) p+\frac{1}{2} \sigma(t, x, u) \sigma^{\top}(t, x, u) P
$$

denotes the generalized Hamiltonian. We will denote

$$
\bar{u}(t, x, p, P) \in \underset{u \in U}{\operatorname{argmax}} G(t, x, u, p, P) .
$$

\section{Necessary Conditions}

In this section we deduce a PDE, that an optimal control must satisfy, as an alternative to the HJB equation. Our derivation depends on the application of the stochastic maximum principle (MP hereafter). The MP and technical conditions on functions $f, \sigma$ and $S$ that allow for its application can be found in [8]. We will take these conditions for granted in the derivation of the quasilinear PDE as a necessary condition for optimality.

Let $(X, u)$ be an optimal control pair, with $u(t)=\phi(t, X(t))$. Applying the stochastic MP, there are unique square integrable processes $p$ and $q$ that satisfy the backward adjoint equation

$$
\begin{aligned}
& d p(s)=-H_{X}(s, X(s), \phi(s, X(s)), p(s), q(s)) d s+q(s) d w(s), \quad s \in[t, T], \\
& p(T)=S_{X}(T, X(T)),
\end{aligned}
$$

such that the following maximization condition

$$
H(s, X(s), \phi(s, X(s)), p(s), q(s))=\max _{u \in U} H(s, X(s), u, p(s), q(s))
$$

holds for every $s \in[t, T]$, P a.s., where $H(t, x, u, p, q)=f(t, x, u) p+\sigma(t, x, u) q^{\top}$ is the stochastic Hamiltonian.

Definition 3.1 (Adjoint feedback) A function $\gamma:[0, T] \times \mathbb{R} \rightarrow \mathbb{R}$ is called an adjoint feedback iff it expresses the adjoint process $p$ in terms of the state variable $X, p(s)=$ $\gamma(s, X(s))$.

To facilitate the exposition of our results, throughout the paper we impose the following: At the optimal $\widehat{\phi}$

$$
\forall(t, x) \in[0, T] \times \mathbb{R}, \quad f_{u}(t, x, \widehat{\phi}) \neq 0 \quad \text { and } \quad\left(\sigma_{u} \sigma^{\top}\right)(t, x, \widehat{\phi}) \neq 0 .
$$


These conditions imply that both $\gamma$ and $\gamma_{x}$ are different from zero, as can easily be seen in the proof of the next result. Thus, the function $F$ defined on $[0, T] \times \mathbb{R} \times U$ by

$$
F(t, x, u)=-\frac{f_{u}}{\sigma_{u} \sigma^{\top}}(t, x, u),
$$

makes sense in a neighborhood of $\widehat{\phi}$.

In the next proposition we show that, under suitable conditions, the adjoint feedback must satisfy a second order quasilinear PDE. The special structure is due to the assumption that the state variable is one-dimensional.

Proposition 3.1 Suppose that $\gamma$ be an adjoint feedback, continuous on $[0, T] \times \mathbb{R}$, of class $C^{1,2}$ on $[0, T) \times \mathbb{R}$ and that $G(t, x, u, p, P)$ be of class $C^{1}$ with respect to all the variables. Then, the adjoint feedback $\gamma$, almost everywhere, satisfies the PDE

$$
\gamma_{t}+\frac{\partial}{\partial x} \max _{u \in U} G\left(t, x, u, \gamma, \gamma_{x}\right)=0
$$

with terminal condition

$$
\gamma(T, x)=S_{x}(T, x) .
$$

Proof We omit the arguments of the functions in several parts of the proof to simplify notation. Applying Itô's formula to $\gamma$ we get

$$
d p(s)=\left(\gamma_{s}+f \gamma_{x}+\frac{1}{2} \sigma \sigma^{\top} \gamma_{x x}\right) d s+\sigma \gamma_{x} d w(s), \quad s \in[t, T],
$$

and equating the volatility terms of (4) and (10)

$$
q=\sigma \gamma_{x}
$$

Next, equating the drift terms of (4) and (10),

$$
\begin{aligned}
\gamma_{t}+f \gamma_{x}+\frac{1}{2} \sigma \sigma^{\top} \gamma_{x x} & =-H_{x}=-f_{x} \gamma-\sigma_{x} q^{\top} \\
& =-f_{x} \gamma-\sigma_{x} \sigma^{\top} \gamma_{x} .
\end{aligned}
$$

Now we consider $\bar{G}\left(t, x, \gamma, \gamma_{x}\right)=\max _{u \in U} G\left(t, x, u, \gamma, \gamma_{x}\right)$, the maximum of $G$ with respect to $u \in U$. By Danskin's Theorem, [9], $\bar{G}$ is differentiable a.e. with respect to $x$, and the derivative at points where it exists is

$$
\frac{\partial}{\partial x} \bar{G}\left(t, x, \gamma, \gamma_{x}\right)=f_{x} \gamma+f \gamma_{x}+\sigma_{x} \sigma^{\top} \gamma_{x}+\left.\frac{1}{2} \sigma \sigma^{\top} \gamma_{x x}\right|_{u=\bar{u}\left(t, x, \gamma, \gamma_{x}\right)} .
$$

Thus, (11) can be rewritten as

$$
\gamma_{t}+\frac{\partial}{\partial x} \bar{G}\left(t, x, \gamma, \gamma_{x}\right)=0
$$


which is the PDE stated in the proposition. Finally, the terminal condition (9) is a consequence of the transversality condition of the MP, (5), and that $\gamma$ is an adjoint feedback, thus $\gamma(T, X(T))=p(T)=S_{X}(T, X(T))$.

Remark 3.1 One aspect that may make PDE (8) impractical is that it depends on the maximizer $\bar{u}$ which is, in general, not known. To get an explicit PDE we will impose the condition that the optimal feedback must be interior to $U$. Note that, even in this case, the PDE for the adjoint feedback continues to be non-explicit. However, the PDE for $\phi$ will always be explicit-see Remark 3.2 below-and holds for any onedimensional control problem of Mayer type fulfilling the conditions imposed in this paper-i.e. smoothness and interiority of the optimal control-.

Next theorem shows the PDE that the optimal $\phi$ satisfies.

Theorem 3.1 Suppose that $\gamma$ be an adjoint feedback and $\phi \in \mathcal{U}$ be the unique admissible interior optimal Markov control of the problem (1), (2), continuous on $[0, T] \times \mathbb{R}$, and of class $C^{1,2}$ on $[0, T) \times \mathbb{R}$. Then, $\phi$ satisfies the quasilinear PDE of second order

$$
\begin{aligned}
& \frac{\partial}{\partial t} F(t, x, \phi)+\frac{\partial}{\partial x} G\left(t, x, \phi, F(t, x, \phi), F^{2}(t, x, \phi)\right) \\
& +\frac{\partial^{2}}{\partial x^{2}} G(t, x, \phi, 1, F(t, x, \phi))=0,
\end{aligned}
$$

with terminal condition

$$
S_{x}(T, x) F(T, x, \phi(T, x))=S_{x x}(T, x) .
$$

Proof Since the argument maximizing $H$ is interior to $U,(6)$ implies

$$
H_{u}(s, X(s), \phi(s, X(s)), p(s), q(s))=0, \quad \forall s \in[t, T], \quad \text { P-a.s., }
$$

that is

$$
f_{u} p+\sigma_{u} q^{\top}=0
$$

Hence, since $q=\sigma \gamma_{x}$ was shown in the proof of Proposition 3.1 and recalling the definition of $F$ in (7), the following equality holds:

$$
\gamma(t, x) F(t, x, \phi(t, x))=\gamma_{x}(t, x) \text {. }
$$

We omit the arguments of the functions in some parts of the proof, when no confusion arises. We will go through the proof in the following steps.

1. Divide the PDE (8), $\gamma_{t}+(\partial / \partial x) G=0$, by $\gamma$ and notice that

$$
\frac{\partial}{\partial x} \frac{G}{\gamma}=\frac{1}{\gamma} \frac{\partial}{\partial x} G-\frac{\gamma_{x}}{\gamma^{2}} G
$$


to get

$$
\frac{\gamma_{t}}{\gamma}+\frac{\gamma_{x}}{\gamma^{2}} G+\frac{\partial}{\partial x} \frac{G}{\gamma}=0
$$

2. By definitions of $G$ and (15)

$$
\begin{aligned}
& \frac{\gamma_{x}}{\gamma^{2}} G\left(t, x, \phi, \gamma, \gamma_{x}\right)=\left.\left(f F+\frac{1}{2} \sigma \sigma^{\top} F^{2}\right)\right|_{(t, x, \phi)}=G\left(t, x, \phi, F, F^{2}\right), \\
& \frac{1}{\gamma} G\left(t, x, \phi, \gamma, \gamma_{x}\right)=\left.\left(f+\frac{1}{2} \sigma \sigma^{\top} F\right)\right|_{(t, x, \phi)}=G(t, x, \phi, 1, F) .
\end{aligned}
$$

3. Take the derivative of (16) with respect to $x$ and then substitute the expressions in step 2 to find

$$
\frac{\partial}{\partial x} \frac{\gamma_{t}}{\gamma}+\frac{\partial}{\partial x} G\left(t, x, \phi, F, F^{2}\right)+\frac{\partial^{2}}{\partial x^{2}} G(t, x, \phi, 1, F)=0
$$

4. Finally, note that $(\partial / \partial x)\left(\gamma_{t} / \gamma\right)=(\partial / \partial t)\left(\gamma_{x} / \gamma\right)=F_{t}$ because $\gamma$ is of class $C^{1,2}$. Using this fact in the above equation, we get (13).

The final condition (14) is obtained as follows. By the transversality condition (9), and the equality $p(T)=\gamma(T, X(T))$ we get $\gamma(T, X(T))=S_{X}(T, X(T))$. Plugging this into the expression for $F$ given in (15) and renaming $X(T)=x$ we get (14).

Remark 3.2 Note that $F$ is always an explicit expression of $(t, x, u)$; thus (13) depends only on $t, x$, and $\phi(t, x)$, once

$$
F(t, x, \phi)=-\frac{f_{u}(t, x, \phi)}{\left(\sigma_{u} \sigma^{\top}\right)(t, x, \phi)}
$$

is substituted throughout in (13). Then, a PDE only involving the unknown control $\phi(t, x)$ is obtained, once we expand the derivatives (see the Appendix):

$$
\begin{gathered}
\phi_{t}(t, x)+A(t, x, \phi(t, x)) \phi_{x}(t, x)+B(t, x, \phi(t, x)) \phi_{x}^{2}(t, x) \\
+C(t, x, \phi(t, x)) \phi_{x x}(t, x)+D(t, x, \phi(t, x))=0 .
\end{gathered}
$$

The coefficients are (we omit the arguments of the functions below to simplify notation):

$$
\begin{aligned}
A= & f+2 \sigma_{x} \sigma^{\top}+\sigma \sigma^{\top} F+\left(f_{x u}+\left(\sigma_{x u}+\sigma_{x} \sigma_{u}^{\top}\right) F+\sigma_{u} \sigma^{\top} F_{x}+\sigma \sigma^{\top} F_{x u}\right) / F_{u}, \\
B= & \sigma_{u} \sigma^{\top}+\sigma \sigma^{\top} F_{u u} /\left(2 F_{u}\right), \\
C= & \sigma \sigma^{\top} / 2, \\
D= & \left(f_{x x}+\left(\sigma_{x x} \sigma^{\top}+\sigma_{x} \sigma_{x}^{\top}\right) F+F_{t}+\left(f+2 \sigma_{x} \sigma^{\top}+\sigma \sigma^{\top} F\right) F_{x}+\sigma \sigma^{\top} F_{x x} / 2\right. \\
& \left.+\left(f_{x} F+\sigma_{x} \sigma^{\top} F^{2}\right)\right) / F_{u},
\end{aligned}
$$


where we suppose $F_{u} \neq 0$. Thus, the PDE (17) is of quasilinear type, that is, the second order derivative $\phi_{x x}$ appears linearly with a factor, $\left(\sigma^{\top} \sigma\right)(t, x, \phi)$, depending on the solution itself. The theoretical advantage of this PDE over the HJB equation is that the HJB equation is nonlinear in the second order derivative $V_{x x}$.

\section{Value Function and Sufficient Conditions}

Before proceeding to establish sufficient conditions for optimality in this section, in the following definition we give a weak notion of a solution of the PDE (13). The reason is that, for our purposes, it suffices to consider $C^{1,1}$ solutions.

Definition 4.1 A function $\phi$ is a $C^{1,1}$ solution of the Cauchy problem (13), (14), iff it satisfies the integral equation

$$
\frac{\partial}{\partial t} \int_{x_{1}}^{x_{2}} F^{\phi}(t, z) d z+L^{\phi}\left(t, x_{2}\right)-L^{\phi}\left(t, x_{1}\right)=0, \quad x_{1}, x_{2} \in \mathbb{R},
$$

and the final condition

$$
S_{x}(T, x) F(T, x, \phi(T, x))=S_{x x}(T, x) .
$$

We have used the short-hand

$$
\begin{aligned}
& F^{\phi}(t, x)=F(t, x, \phi(t, x)), \\
& L^{\phi}(t, x)=G\left(t, x, \phi, F^{\phi},\left(F^{\phi}\right)^{2}\right)+\frac{\partial}{\partial x} G\left(t, x, \phi, 1, F^{\phi}\right) .
\end{aligned}
$$

In this section we show that a solution $\widehat{\phi}$ of class $C^{1,1}$ of (18) and (19), maximizing the generalized Hamiltonian for all $(t, x)$, is a solution of the stochastic control problem (1), (2). We also find the connection between the optimal control, the adjoint feedback, and the value function.

A proposition gives the adjoint feedback in terms of a solution to (18), (19). It is important to obtain an explicit expression for $\gamma$, since it is the derivative with respect to $x$ of the value function, and consequently has the economic interpretation of a "shadow price", that is, if $X$ represents the stock of a given resource and $V$ is the optimal profit earned during $[0, T]$, then $\gamma$ is the maximum price that would be worth paying for one additional unit of the resource. The adjoint variable $\gamma$ is also the state variable of the dual control problem associated to the original problem, see e.g. [10] or [11]. In our framework, the value function is differentiable because the optimal policy is smooth and interior to the control region. Furthermore, once $\gamma$ is known, it is straightforward to obtain the value function, as will be shown in Theorem 4.1 below.

Proposition 4.1 Let $\widehat{\phi}$ be an admissible control of class $C^{1,1}$ satisfying (18), (19). Then, for any $t \in[0, T]$ and $x \in \mathbb{R}$ and any $\alpha \in \mathbb{R}$, the adjoint feedback $\gamma$ is of class $C^{1,2}$ and is given by

$$
\gamma(t, x)=S_{x}(T, \alpha) e^{\int_{t}^{T} L^{\widehat{\phi}}(s, \alpha) d s} e^{\int_{\alpha}^{x} F^{\widehat{\phi}}(t, z) d z} .
$$


Proof It is clear that $\gamma$, as given by (20), has the required smoothness. Taking the derivative in (20) with respect to $t$ we get

$$
\gamma_{t}(t, x)=\gamma(t, x)\left(\frac{\partial}{\partial t} \int_{\alpha}^{x} F^{\widehat{\phi}}(t, z) d z-L^{\widehat{\phi}}(t, \alpha)\right) .
$$

Since $F$ satisfies (18), then selecting $x_{1}=\alpha$ and $x_{2}=x$

$$
\frac{\partial}{\partial t} \int_{\alpha}^{x} F^{\widehat{\phi}}(t, z) d z-L^{\widehat{\phi}}(t, \alpha)=-L^{\widehat{\phi}}(t, x) .
$$

Substituting into (21), we have

$$
\gamma_{t}(t, x)+\gamma(t, x) L^{\widehat{\phi}}(t, x)=0
$$

Then, using the identities in steps 1 and 2 of the proof of Theorem 3.1 we have:

$$
\begin{aligned}
\gamma L^{\widehat{\phi}} & =\gamma G\left(t, x, \phi, F^{\phi},\left(F^{\phi}\right)^{2}\right)+\gamma \frac{\partial}{\partial x} G\left(t, x, \phi, 1, F^{\phi}\right) \\
& =\frac{\partial}{\partial x} G\left(t, x, \bar{u}\left(t, x, \gamma, \gamma_{x}\right), \gamma, \gamma_{x}\right)
\end{aligned}
$$

where $\bar{u}$ is defined in (3). Thus, by (22), the expression defined in (20) satisfies (8). The final condition (9) follows from (14):

$$
\gamma(T, x)=S_{x}(T, \alpha) e^{\int_{\alpha}^{x} F^{\widehat{\phi}}(T, z) d z}=S_{x}(T, \alpha) e^{\ln \left|S_{x}(T, x) / S_{x}(T, \alpha)\right|}=S_{x}(T, x) .
$$

Finally, the independence of $\gamma$ from the constant $\alpha$ is deduced by verifying that the derivative of $\gamma$ with respect to $\alpha$ is zero. This is clear if $S_{x}(t, \alpha)=0$; so suppose that $S_{x}(T, \alpha) \neq 0$. Then

$$
\begin{aligned}
\frac{\partial}{\partial \alpha} \gamma(t, x) & =\gamma(t, x)\left(\frac{S_{x x}(T, \alpha)}{S_{x}(T, \alpha)}-F^{\widehat{\phi}}(t, \alpha)+\int_{t}^{T} L_{x}^{\widehat{\phi}}(s, \alpha) d s\right) \\
& =\gamma(t, x)\left(\int_{t}^{T} F_{t}^{\widehat{\phi}}(s, \alpha) d s+\int_{t}^{T} L_{x}^{\widehat{\phi}}(s, \alpha) d s\right)=0,
\end{aligned}
$$

where the second equality holds by (14) and the last equality is implied by (13).

Given a solution $\widehat{\phi}$ of (18), (19), Proposition 4.1 shows that an adjoint feedback $\gamma$ exists. From this information we construct the value function $V$. To simplify the notation, for $u \in U$, we define

$$
\mathcal{G}^{u}(t, x)=G\left(t, x, u, \gamma(t, x), \gamma_{x}(t, x)\right) \text {. }
$$

Theorem 4.1 (Value function) Let $\widehat{\phi}$ be an admissible control solution of (18), (19), such that

$$
\forall(t, x) \in[0, T] \times \mathbb{R}, \forall u \in U, \quad \mathcal{G}^{\widehat{\phi}}(t, x) \geq \mathcal{G}^{u}(t, x)
$$


Then for an arbitrary constant $\alpha, W$ given by

$$
W(t, x)=e^{-\delta(T-t)}\left(\int_{\alpha}^{x} \gamma(t, z) d z+\int_{t}^{T} \mathcal{G}^{\widehat{\phi}}(s, \alpha) d s+S(T, \alpha)\right)
$$

is a $C^{1,3}$ solution of the HJB equation and satisfies $W(T, x)=S(T, x)$. Moreover, if for all $x$

$$
|\gamma(t, x)| \leq K_{1}\left(1+|x|^{K_{2}}\right)
$$

for some constants $K_{1}$ and $K_{2}>-1$, then $W=V$ is the value function, and $\widehat{\phi}$ is an optimal control.

Proof It is obvious that $W$, defined in (24), is a function of class $C^{1,3}$, with $W_{x}=$ $e^{-\delta(T-t)} \gamma(t, x)$ and $W_{x x}=e^{-\delta(T-t)} \gamma_{x}(t, x)$, since we know $\gamma \in C^{1,2}$ by Proposition 4.1. Integrating (12) with respect to $x$ and interchanging the order of the integration and derivation operations, we have

$$
\frac{\partial}{\partial t} \int_{\alpha}^{x} \gamma(t, z) d z+\mathcal{G}^{\widehat{\phi}}(t, x)-\mathcal{G}^{\widehat{\phi}}(t, \alpha)=0 .
$$

Taking the derivative with respect to $t$ in (24), we find:

$$
e^{\delta(T-t)}\left(-\delta W(t, x)+W_{t}(t, x)\right)=\frac{\partial}{\partial t} \int_{\alpha}^{x} \gamma(t, z) d z-\mathcal{G}^{\widehat{\phi}}(t, \alpha)=-\mathcal{G}^{\widehat{\phi}}(t, x) .
$$

Hence, by definition of $\mathcal{G}^{\widehat{\phi}}(t, x)$

$$
W_{t}(t, x)+W_{x}(t, x) f(t, x, \widehat{\phi}(t, x))+\frac{1}{2}\left(\sigma \sigma^{\top}\right)(t, x, \widehat{\phi}(t, x)) W_{x x}(t, x)=\delta W(t, x) .
$$

On the other hand, by assumption (23)

$$
\begin{gathered}
W_{x}(t, x) f(t, x, \widehat{\phi}(t, x))+\frac{1}{2} W_{x x}(t, x)\left(\sigma \sigma^{\top}\right)(t, x, \widehat{\phi}(t, x)) \\
\geq G\left(t, x, u, W_{x}(t, x), W_{x x}(t, x) \sigma(t, x, u)\right), \quad \forall u \in U .
\end{gathered}
$$

In consequence, (26) and (27) imply that $W$ satisfies the HJB equation. The final condition also holds, since

$$
W(T, x)=\int_{\alpha}^{x} \gamma(T, z) d z+S(T, \alpha)=\int_{\alpha}^{x} S_{x}(T, z) d z+S(T, \alpha)=S(T, x),
$$

due to (5). To complete the proof of the first part of the theorem, it is immediate to check that (24) does not depend on $\alpha$, as this was done in Proposition 4.1.

Finally, if $\gamma$ satisfies (25), then by (24) $W$ is polynomially bounded. Hence, to make $W$ the value function and $\widehat{\phi}$ truly optimal, it suffices to apply the verification theorem in [7]. 
Remark 4.1 Condition (23) automatically holds when $\widehat{\phi}$ is interior to the control set $U$ and for all $(t, x) \in[0, T] \times \mathbb{R}$ the function $G\left(t, x, u, \gamma, \gamma_{x}\right)$ is concave with respect to $u$, since the equality

$$
G_{u}\left(t, x, \widehat{\phi}, \gamma(t, x), \gamma_{x}(t, x)\right)=H_{u}\left(t, x, \widehat{\phi}, \gamma(t, x), \sigma(t, x, \widehat{\phi}) \gamma_{x}(t, x)\right)=0
$$

is satisfied trivially for any smooth solution $\widehat{\phi}$ of problem (13), (14); this means that $\widehat{\phi}$ is a critical point of the concave function $u \mapsto G(\ldots, u, \ldots)$, hence $\widehat{\phi}$ is a global maximum of $G(\ldots, u, \ldots)$.

\section{Application to Models with Linear Dynamics}

We now show the form of (13) in next two examples.

\subsection{General Problem}

Consider a control problem with linear drift

$$
f(t, x, u)=a(t) x+b(t) u
$$

and linear diffusion coefficient

$$
\sigma(t, x, u)=\left(c_{1}(t) x+d_{1}(t) u, \ldots, c_{\ell}(t) x+d_{\ell}(t) u\right),
$$

where the time-dependent vectors $c(t)=\left(c_{i}(t)\right)_{i=1}^{\ell}$ and $d(t)=\left(d_{i}(t)\right)_{i=1}^{\ell}$ are differentiable, with $b(t) \neq 0$ and $d(t) d^{\top}(t)>0$ for every $t \in[0, T]$. In the following we drop the time dependence from the notation. The definition of $F$ in (7) gives

$$
F(x, u)=-\frac{b}{c d^{\top} x+d d^{\top} u}, \quad \text { or } \quad u=\bar{u}(t, x, F)=-\left(\frac{b}{d d^{\top}}\right) F^{-1}-\left(\frac{c d^{\top}}{d d^{\top}}\right) x .
$$

Obviously, for this particular class of models it is always possible to find $\bar{u}$. Hence, the PDE (8) satisfied by $\gamma$ can be explicitly found (we omit the arguments in the functions)

$$
\gamma_{t}+\frac{\partial}{\partial x}\left(\left(a-b \frac{c d^{\top}}{d d^{\top}}\right) x \gamma+\frac{1}{2}\left(c c^{\top}-\frac{\left(c d^{\top}\right)^{2}}{d d^{\top}}\right) x^{2} \gamma_{x}-\frac{1}{2} \frac{b^{2}}{d d^{\top}} \frac{\gamma^{2}}{\gamma_{x}}\right)=0,
$$

because

$$
G\left(t, x, u, \gamma, \gamma_{x}\right)=(a x+b u) \gamma+\frac{1}{2}\left(c c^{\top} x^{2}+2 c d^{\top} x u+d d^{\top} u^{2}\right) \gamma_{x} .
$$

Moreover, an explicit PDE for $F=\gamma_{x} / \gamma$ arises from (13)

$$
F_{t}+\frac{1}{2} \frac{\partial}{\partial x}\left(2 \bar{a}(t) x F+\bar{c}(t) x^{2} F^{2}\right)+\frac{1}{2} \frac{\partial^{2}}{\partial x^{2}}\left(-\bar{b}(t) F^{-1}+\bar{c}(t) x^{2} F\right)=0,
$$


where

$$
\bar{a}(t)=a(t)-b(t) \frac{c d^{\top}(t)}{d d^{\top}(t)}, \quad \bar{b}(t)=\frac{b^{2}(t)}{d d^{\top}(t)}, \quad \bar{c}(t)=c c^{\top}(t)-\frac{\left(c d^{\top}(t)\right)^{2}}{d d^{\top}(t)} .
$$

Substituting $F$ in (29) with its expression in terms of $\phi$

$$
F(t, x, \phi)=-\frac{b(t)}{c(t) d^{\top}(t) x+d d^{\top}(t) \phi},
$$

an explicit PDE for $\phi$ is obtained. The PDE for $\phi$ will be shown for the Merton problem in next section, in order to save space.

We shall now make a few remarks on the above PDEs.

1. Equation (28) has already been obtained in the financial literature in the case $c(t)=0$ for all $t \in[0, T]$ and $\ell=1$, see e.g. [12]. See also Sect. 5.2 below, where we study Merton's problem with deterministic coefficients. It is also known that, under these conditions, $c=0$ and $\ell=1$, the hodograph transform $X(t, \gamma(t, x))=x$ (that is, for each $t, X(t, \cdot)$ is the inverse of function $\gamma$ ) linearizes (28) to the PDE

$$
X_{t}-\left(a-\frac{b c}{d}\right) X-\left(a-\frac{b c}{d}-\frac{b^{2}}{d^{2}}\right) \gamma X_{\gamma}+\frac{b^{2}}{2 d^{2}} \gamma^{2} X_{\gamma \gamma}=0
$$

with final condition $X(T, \gamma)=\left(S^{\prime}\right)^{-1}(\gamma)$, see [12] or [5]. Under suitable conditions, assuring that $X$ is a global $C^{2}$ diffeomorphism for each $t$, the problem can be solved fairly well, and explicit expressions for the optimal control and the value function can be recovered. The same results can also be obtained by the martingale approach, see [13]. However, the hodograph transform does not linearize the PDE for $\gamma$ in the general case with non-null vector $c$ and $\ell>1$ Brownian motions. An interesting question is to find a suitable transformation (if any) that works in this case.

2. For any constant $\rho, F=-\rho / x$ is a (stationary) solution of (29). This solution is consistent with a parametric family of objective functions $S$, of HARA (Hyperbolic Absolute Risk Aversion) type, $S(T, x)=\frac{K}{1-\rho} x^{1-\rho}$, when $\rho>0, \rho \neq 1$ or $S(T, x)=K \ln x$ if $\rho=1$, in both cases with $K>0$. Note that $F$ satisfies the final condition (14) and $S$ is strictly concave. From Theorem 3.1, the linear control (in variable $x$ )

$$
\phi(t, x)=\left(d(t) d^{\top}(t)\right)^{-1}\left(\rho^{-1} b(t)+c(t) d^{\top}(t)\right) x
$$

is a solution of (13) and satisfies the final condition (14); therefore, it is a candidate for an optimal control of this family of problems. In the following section we give sufficient conditions for a solution of the PDE for the control, based on Theorem 4.1, to be actually an optimal control.

3. Knowledge of the PDE (13) allows us to address the inverse or integrability problem, which consists of recovering the utility function $S$ from a given optimal investment control $u$. We analyze this problem here, for linear controls. The problem consists in determining an increasing and strictly concave function $S$ such 
that $\phi(t, x)=m(t) x+n(t)$, with given smooth functions $m$ and $n$, is the solution of the control problem, for suitable functions $a, b, c$ and $d$, as well as of the dimension of the Brownian vector, $\ell$. By (7)

$$
F^{\phi}(t, x)=F(t, x, \phi(t, x))=-\frac{b(t)}{c(t) d^{\top}(t) x+d(t) d^{\top}(t)(m(t) x+n(t))}
$$

must be a solution to (29), and the final condition (14) holds.

Let $\mu(t)=c(t) d^{\top}(t)+d(t) d^{\top}(t) m(t), \beta(t)=d(t) d^{\top}(t) n(t)$, and recall the definition of functions $\bar{a}(t)$ and $\bar{c}(t)$ given above. Substituting (30) into (29) we get the conditions (we omit the time argument)

$$
\begin{aligned}
& \dot{b} \mu^{2}-b \mu \dot{\mu}=0, \\
& 2 \dot{b} \mu \beta-b \dot{\mu} \beta-\mu b \dot{\beta}+\mu b \bar{a} \beta-b^{2} \bar{c} \beta=0, \\
& (\dot{b} \beta-b \dot{\beta}+b \bar{a} \beta+b \bar{c} \beta) \beta=0 .
\end{aligned}
$$

As above, we suppose $b(t) \neq 0$ and $d(t) d^{\top}(t)>0$ for all $t$, and consider functions $c, d$ such that $\mu(t) \neq 0$ for all $t$. We distinguish two cases.

(a) $n(t) \neq 0$ for all $t$. Then, from (31) $\mu(t)=k b(t)$ for some constant $k$. Hence

$$
m(t)=\frac{k b(t)-c(t) d^{\top}(t)}{d(t) d^{\top}(t)}
$$

must hold. On the other hand, (33) reduces to

$$
\dot{\beta}(t)=\beta(t)\left(\frac{\dot{b}(t)}{b(t)}+\bar{a}(t)+\bar{c}(t)\right),
$$

hence $\beta(t)=-\beta(T) \exp \left\{\int_{t}^{T}(\dot{b}(s) / b(s)+\bar{a}(s)+\bar{c}(s)) d s\right\}$ and

$$
\begin{aligned}
n(t) & =\frac{-\beta(T)}{d d^{\top}(t)} \exp \left(\int_{t}^{T}(\dot{b}(s) / b(s)+\bar{a}(s)+\bar{c}(s)) d s\right) \\
& =\frac{-\beta(T) b(T)}{d d^{\top}(t) b(t)} \exp \left(\int_{t}^{T}(\bar{a}(s)+\bar{c}(s)) d s\right) .
\end{aligned}
$$

There are multiple selections of functions $a, b, c$ and $d$ such that both (34) and (35) hold. Plugging $\mu(t)=k b(t)$ into (32) leads to

$$
b \dot{b} k \beta-k b^{2} \dot{\beta}+k b^{2} \bar{a} \beta-\bar{c} \beta b^{2}=0
$$

so by using (33) one obtains $-(1+k) \bar{c}(t) b^{2}(t) \beta(t)=0$ for all $t$. This implies $k=-1$ or $\bar{c}(t)=0$, because $b(t) \neq 0, \beta(t) \neq 0$ for all $t$. Note that $\bar{c}(t)=0$ holds if $\ell=1$.

To determine function $S$, we use the final condition (14). From the identity

$$
-S^{\prime}(x) \frac{b(T)}{\mu(T) x+\beta(T)}=S^{\prime \prime}(x),
$$


it is not difficult to find, with $k=\mu(T) / b(T)$, that

$$
\begin{aligned}
S(x) & =\frac{-K}{(1-k) b(T)}(\mu(T) x+\beta(T))^{-(1-k) / k}, \quad \text { if } k \neq 1, \\
& =\frac{K}{b(T)} \ln (\mu(T) x+\beta(T)), \quad \text { if } k=1,
\end{aligned}
$$

where the constants $K$ and $k$ are both strictly positive. In this way, $S$ is strictly increasing. Moreover, choosing $b(T)<0$, it is also strictly concave. The case with $\mu(T)=0, \beta(T) \neq 0$ is also possible, with $S(x)=$ $-K(\beta(T) / b(T)) \exp \{(-b(T) / \beta(T)) x\}, K>0$ and $b(T) / \beta(T)>0$.

(b) $n(t)=0$ for some $t \in[0, T]$. Then $n$ must actually be identically null because $\beta(t)=0$ for all $t$. This is a consequence of supposing $b(t) \neq 0, \mu(t) \neq 0$ and $d(t) d^{\top}(t)>0$ for all $t$. Thus, the only constraint that appears is $\mu(t)=k b(t)$ for some constant $k$. The form of $S$ can be recovered from (36) with $\beta(T)=0$ and $\mu(T) \neq 0$.

\subsection{Merton's Problem with Deterministic Coefficients}

The problem in Sect. 5.1 above encompasses, in particular, a variant of the investment problem of Merton [14], where there is no running utility from consumption, and where the objective is to maximize the utility of terminal wealth. That is, an investor wants to maximize the expected utility $S$ of the final wealth at a fixed date $T$. Along the time interval $[0, T]$ the decision agent invests in two assets, one of them a risky asset whose price, $P^{1}$, evolves according to the SDE

$$
d P^{1}(t)=(b(t)+r(t)) P^{1}(t) d t+\sigma(t) P^{1}(t) d w(t), \quad P^{1}(0) \text { known, }
$$

and the other is a bank account, $P^{0}$, which is driven by

$$
d P^{0}(t)=r(t) P^{0}(t) d t, \quad P^{0}(0)=1,
$$

where $r, b$ and $\sigma$ are positive, deterministic functions of time.

Let $u(s)$ be the amount of wealth invested in the risky asset at time $s$, and let $X(s)-u(s)$ be the amount invested in the bond, where $X(s)$ is the accumulated wealth until time $s$. Then, $X(s)$ satisfies the SDE

$$
\begin{aligned}
& d X(s)=(r(s) X(s)+b(s) u(s)) d t+\sigma(s) u(s) d w(s), \quad t \leq s \leq T, \\
& X(t)=x, \quad x \geq 0 .
\end{aligned}
$$

Given wealth's level $X(t)=x$ at date $t \in[0, T]$, the problem is to choose an investment policy $u$ solving the problem

$$
\max _{u \in \mathcal{U}(t, x)} e^{-\delta(T-t)} \mathrm{E}\{S(X(T)) \mid X(t)=x\}=\max _{u \in \mathcal{U}(t, x)} e^{-\delta(T-t)} \mathrm{E}_{x} S(X(T)),
$$

subject to (37), where $S$ is a strictly increasing and strictly concave utility function. This model has been well studied in the literature. Merton's problem is obtained from 
the example in Sect. 5.1 by selecting $a=r, c=0$ and $d=\sigma$, with $\ell=1$. It is easy to compute (13) and the final condition (14)

$$
\begin{aligned}
& F_{t}+r(t) \frac{\partial}{\partial x}(x F)-\frac{1}{2} b(t) \theta(t) \frac{\partial^{2}}{\partial x^{2}}\left(\frac{1}{F}\right)=0, \\
& F(T, x, \phi(T, x))=\frac{S^{\prime \prime}(x)}{S^{\prime}(x)},
\end{aligned}
$$

where $\theta(t)=b(t) / \sigma^{2}(t)$. Then, the PDE for $\phi=-\theta(t) / F$ is

$$
\frac{\partial}{\partial t}\left(-\frac{\theta(t)}{\phi(t, x)}\right)-r(t) \theta(t) \frac{\partial}{\partial x}\left(\frac{x}{\phi(t, x)}\right)+\frac{1}{2} b(t) \frac{\partial^{2}}{\partial x^{2}} \phi(t, x)=0 .
$$

Taking the derivatives we get the Cauchy problem in $(t, x) \in[0, T] \times[0, \infty)$

$$
\begin{aligned}
& \phi_{t}-r(t)\left(\phi-x \phi_{x}\right)-\frac{\dot{\theta}(t)}{\theta(t)} \phi+\frac{1}{2} \sigma^{2}(t) \phi^{2} \phi_{x x}=0 \quad t<T, x>0, \\
& \phi(T, x)=\theta(T) R(x), \quad x>0 \\
& \phi(t, 0)=0, \quad t<T .
\end{aligned}
$$

Here, $R(x)=-S^{\prime}(x) / S^{\prime \prime}(x)$ is the absolute risk tolerance index of the decision agent (the inverse of the absolute risk aversion index).

The equation found in [5] is a particular case when constant coefficients are considered. These authors use the equation to study asymptotic properties of the optimal investment control. The solution found in Sect. 5.1 for HARA utilities is $\widehat{\phi}(t, x)=\rho^{-1} \theta(t) x$ with $\rho>0$, which is of course well known in the literature.

Note that, unless $R$ satisfies $R(0)=0$, the initial condition is not compatible with the boundary condition at $x=0$ and the existence of a smooth solution is problematic. Thus, we impose $R(0)=0$ in the following theorem, and show the existence of a solution to the Cauchy problem. We consider utility functions $S$ possibly with unbounded absolute risk tolerance, but which exhibit bounded relative risk tolerance.

Theorem 5.1 Assume that functions $r, b, \sigma, \theta$ and $\dot{\theta}$ be of class $C^{2}$ and bounded, with $\sigma>0, \theta>0$, and that function $R$ be of class $C^{2}$ and satisfies $R(0)=0$, $R(x)>0$ for $x>0, \sup _{x \in[0, \infty)} R(x) / x<\infty$ and $\lim _{x \rightarrow 0^{+}} R(x) / x$ exist. Then, there is a solution of the Cauchy problem (38) of class $C^{1,2}$. Moreover, any solution $\phi$ is globally Lipschitz in $x$ and satisfies for all $t \in[0, T]$

$$
\theta(t) x\left(\inf _{y \in[0, \infty)} \frac{R(y)}{y}\right) \leq \phi(t, x) \leq \theta(t) x\left(\sup _{y \in[0, \infty)} \frac{R(y)}{y}\right) .
$$

Proof Let $v=\phi / x$ and $\tau=T-t$. Then, the Cauchy problem for $v$ is (we omit the arguments in $v$ ) 


$$
\begin{aligned}
& v_{\tau}-r(T-\tau) x v_{x}+\frac{\dot{\theta}(T-\tau)}{\theta(T-\tau)} v-\frac{1}{2} \sigma^{2}(T-\tau) x v^{2}\left(2 v_{x}+x v_{x x}\right)=0, \\
& v(0, x)=\theta(T) \lim _{x \rightarrow 0^{+}} \frac{R(x)}{x} .
\end{aligned}
$$

We rewrite the PDE in divergence form as follows

$$
v_{\tau}-\frac{1}{2} \sigma^{2}(T-\tau) \frac{\partial}{\partial x}\left(x^{2} v^{2} v_{x}\right)=r(T-\tau) x v_{x}+\frac{\dot{\theta}(T-\tau)}{\theta(T-\tau)} v-\sigma^{2}(T-\tau) x^{2} v v_{x}^{2}
$$

The equation fulfills all the requirements of Theorem 8.1 in Chap. V in [15] (including compatibility between the final and the boundary condition at $x=0$ ), except for the uniform parabolic condition in the second order term. However, the solution never vanishes; thus the equation is truly parabolic in $[0, T] \times(0, \infty)$. This can be seen as follows (we adopt here a device used in [16]). Consider the interval $[1 / n, n]$ and the solution $v_{n}$ in $[0, T] \times[1 / n, n]$ satisfying $v_{n}(\tau, 1 / n)=\theta(T-\tau) n R(1 / n)$, $v_{n}(\tau, n)=\theta(T-\tau) R(n) / n$. Such a solution exists and is of class $C^{1,2}$, with Hölder regularity on the derivatives, see [15]. Let $m_{n}(\tau)=\min _{y \in[1 / n, n]} v_{n}(\tau, y)$. By Danskin's Theorem, [9], function $m_{n}$ is differentiable a.e., and at points of differentiability $\dot{m}_{n}(\tau)=v_{n, \tau}\left(\tau, \xi_{n}(\tau)\right)$, where $v_{n}\left(\tau, \xi_{n}(\tau)\right)=m_{n}(\tau)$.

We will show that $v_{n}(\tau, x)>0$ for all $(\tau, x) \in[0, T] \times(0, \infty)$. Let us suppose, on the contrary, that there exists $\bar{\tau}_{0}$ such that $v_{n}\left(\bar{\tau}_{0}, x\right)=0$ for some $x \in[1 / n, n]$. Then there exists $0<\tau_{0}<\bar{\tau}_{0}$ such that $0<v_{n}\left(\tau_{0}, x\right)<\theta(T-$ $\left.\tau_{0}\right) \max \{n R(1 / n), R(n) / n\}$ and hence the minimum of $v_{n}\left(\tau_{0}, x\right)$ is attained in the interior of $[1 / n, n]$; thus $v_{n, x}\left(\tau, \xi_{n}\left(\tau_{0}\right)\right)=0$. Since $v_{n, x x}\left(\tau, \xi_{n}\left(\tau_{0}\right)\right) \geq 0$, we get $\sigma^{2}(T-\tau)(\partial / \partial x)\left(x^{2} v_{n}^{2} v_{n, x}\right) \geq 0$. Using this information in (39) for $v_{n}$, we get the ordinary differential inequality for $m_{n}$

$$
\dot{m}_{n}(\tau) \geq \frac{\dot{\theta}(T-\tau)}{\theta(T-\tau)} m_{n}(\tau) \quad \text { a.e. } \tau_{0} \leq \tau \leq \bar{\tau}_{0} .
$$

Hence, $m_{n}\left(\bar{\tau}_{0}\right) \geq m_{n}\left(\tau_{0}\right) \theta\left(T-\bar{\tau}_{0}\right) / \theta\left(T-\tau_{0}\right)>0$, arriving to a contradiction. Indeed, the same technique shows that the estimate

$$
v_{n}(\tau, x) \geq m_{n}(\tau) \geq \theta(T-\tau) \inf _{y \in[1 / n, n]} \frac{R(y)}{y}>0
$$

holds. Moreover, for $j<n$ and $x \in[1 / j, j]$

$$
v_{n}(\tau, x) \geq \theta(T-\tau) \inf _{y \in[1 / j, j]} \frac{R(y)}{y}>r_{j}>0
$$

for some constant $r_{j}$ independent of $n$. By a diagonal argument, the solution obtained by the method of [15] is the limit of the sequence $\left\{v_{n}(\tau, x)\right\}_{n \geq 1}$. By (40), the limit satisfies also $v(\tau, x)>0$ for any $t, x>0$. Now, $\phi(t, x)=x v(T-t, x)$ is a solution of the Cauchy problem (38).

For the second part of the theorem, consider $M_{n}(\tau)=\max _{y \in[1 / n, n]} v_{n}(\tau, y)$. A similar reasoning and computation as done above gives $M_{n}(\tau) \leq$ 
$\theta(T-\tau) \sup _{y \in[1 / n, n]} R(y) / y$. Then,

$$
\theta(T-\tau) \inf _{y \in[1 / n, n]} \frac{R(y)}{y} \leq v_{n}(\tau, x) \leq \theta(T-\tau) \sup _{y \in[1 / n, n]} \frac{R(y)}{y} .
$$

Hence, the limit $\phi$ satisfies for all $t \in[0, T]$

$$
\theta(t) x\left(\inf _{y \in[0, \infty)} \frac{R(y)}{y}\right) \leq \phi(t, x) \leq \theta(t) x\left(\sup _{y \in[0, \infty)} \frac{R(y)}{y}\right) .
$$

Next result establishes the existence of a unique solution to the Merton problem when the relative risk aversion index of the agent is bounded by one.

Theorem 5.2 Assume that $S$ be increasing and strictly concave, and that the conditions of the previous theorem hold. Furthermore, suppose that

$$
\bar{R}:=\sup _{x \in[0, \infty)} \frac{R(x)}{x}>1 .
$$

Then, the Cauchy problem (38) admits a unique solution, which is the optimal control of the Merton problem.

Proof To apply Theorem 4.1 it must be shown also that $\phi$ is admissible and that $\gamma$ is polynomially bounded in $x$. The first claim follows since $\phi$ is Lipschitz in $x$; hence a unique strong solution of the SDE exists. For the second claim, note that the dependence of $\gamma$ with respect to $x$ comes, according to (20), from the term

$$
e^{\int_{\alpha}^{x} F^{\widehat{\phi}}(t, z) d z}=e^{-\theta(t) \int_{\alpha}^{x} \frac{1}{\phi(t, z)} d z} .
$$

Since $\theta(t)>0$ and by the previous theorem $\widehat{\phi}(t, x) \leq \bar{R} \theta(t) x$, we have that for any constant $\alpha>0$

$$
e^{\int_{\alpha}^{x} F^{\widehat{\phi}}(t, z) d z} \leq\left(\frac{x}{\alpha}\right)^{-\frac{1}{\bar{R}}}
$$

Thus, for $t$ fixed, $\gamma(t, x)$ satisfies the bound (25) with $K_{2}=-1 / \bar{R}$ since (41) assures $K_{2}>-1$. Next we check that $G\left(t, x, u, \gamma, \gamma_{x}\right)$ is concave in $u$. By (20) $\gamma$ is positive. Hence, it is decreasing in $x>0$ since the derivative

$$
\gamma_{x}(t, x)=F(t, x, \widehat{\phi}(t, x)) \gamma(t, x)=-\frac{b(t)}{\sigma^{2}(t) \widehat{\phi}(t, x)} \gamma(t, x)<0,
$$

because $b(t)>0$ and $\widehat{\phi}(t, x)>0$ for all $t$ and $x>0$. Hence $G_{u u}=\sigma^{2}(t) \gamma_{x}<0$. Then, $\widehat{\phi}$ is optimal by Remark 4.1. Finally, to show uniqueness we will show first that the functional $J(t, x ; u)$ is strictly concave in $u$, and consequently whenever exists, the global maximum is unique a.e. Then, if $\widehat{\phi}^{1}$ and $\widehat{\phi}^{2}$ are two solutions of the Cauchy problem (38) satisfying (41), they are optimal as has been just proved above, hence $\widehat{\phi}^{1}=\widehat{\phi}^{2}$ a.e. 
For $0<\xi<1$, we set $\phi^{\xi}=\xi \phi^{1}+(1-\xi) \phi^{2}$ for admissible $\phi^{1}, \phi^{2}$, with $\phi^{1} \neq \phi^{2}$ on a set of positive measure, and define

$$
\begin{aligned}
& X^{\phi^{\xi}}(\tau)=\int_{t}^{\tau}\left(r(s) X^{\phi^{\xi}}(s)-b(s) \phi^{\xi}(s)\right) d s+\int_{t}^{\tau} \sigma(s) \phi^{\xi}(s) d w(s), \quad X^{\phi^{\xi}}(t)=x, \\
& X^{\xi}=\xi X^{\phi^{1}}+(1-\xi) X^{\phi^{2}} .
\end{aligned}
$$

Note that, by linearity

$$
X^{\xi}(\tau)=\int_{t}^{\tau}\left(r(s) X^{\xi}(s)-b(s) \phi^{\xi}(s)\right) d s+\int_{t}^{\tau} \sigma(s) \phi^{\xi}(s) d w(s) .
$$

Hence, by uniqueness of solution of the SDE, $X^{\phi^{\xi}}=X^{\xi}$ a.s.; thus,

$$
\begin{aligned}
J\left(t, x ; \phi^{\xi}\right) & =e^{-\delta(T-t)} \mathrm{E}_{t x}\left\{S\left(X^{\phi^{\xi}}(T)\right)\right\}=e^{-\delta(T-t)} \mathrm{E}_{t x}\left\{S\left(X^{\xi}(T)\right)\right\} \\
& \left.=e^{-\delta(T-t)} \mathrm{E}_{t x}\left\{S\left(\xi X^{\phi^{1}}(T)\right)+(1-\xi) X^{\phi^{2}}(T)\right)\right\} \\
& >\xi e^{-\delta(T-t)} \mathrm{E}_{t x}\left\{S\left(X^{\phi^{1}}(T)\right)\right\}+(1-\xi) e^{-\delta(T-t)} \mathrm{E}_{t x}\left\{S\left(X^{\phi^{2}}(T)\right)\right\} \\
& =\xi J\left(t, x ; \phi^{1}\right)+(1-\xi) J\left(t, x ; \phi^{2}\right),
\end{aligned}
$$

since $S$ is strictly concave and by linearity of the conditional expectation; thus, $J$ is strictly concave in $u$.

The solution of problem (38) is illustrated in Fig. 1 for a Merton problem subject to a business cycle in the interest rate, which is reflected in $r(t)=0.05+0.02 \sin (\pi t / 2)$. The variance parameter is $\sigma=0.2$, the excess return is $b=0.02$ and the time horizon $T=4$. The utility function is $S(x)=x^{1-\rho_{1}} /\left(1-\rho_{1}\right)+x^{1-\rho_{2}} /\left(1-\rho_{2}\right)$ with $\rho_{1}=0.9$ and $\rho_{2}=3$. The absolute risk tolerance index, $R(x)=10 x \frac{x^{2.1}+1}{9 x^{2.1}+30}$, fits the requirements of Theorem 5.2. In this model, poor people have a relative risk aversion index of approximately 3 , whilst for rich people it is approximately 0.9 . Thus, in agreement with the intuitive sense, rich people are more willing to invest in risky assets than poor people, and the solution is increasing and convex in wealth. The surface gives the optimal amount of money to invest in the risky asset given the date $t$ and the wealth $x$ the agent possesses. The curves $t=$ constant are quite close to each other for $t=0, t=2$ and $t=T=4$, particularly for low values of $x$, which shows that the asset allocation decisions do not depend much on time. Actually, as has been proved in [5], the solution converges as $\tau \rightarrow \infty$ to a stationary solution of the autonomous version of the PDE for $\phi(x)$,

$$
-r\left(\phi-x \phi_{x}\right)+\frac{1}{2} \sigma^{2} \phi^{2} \phi_{x x}=0, \quad x>0, \quad \phi(0)=0 .
$$

For computing the solution, the routine pdepe implemented in MATLAB has been used. 
Fig. 1 Solution profiles and solution surface in the Merton problem with relative risk tolerance index $R(x) / x=10\left(x^{2.1}+1\right) /$ $\left(9 x^{2.1}+30\right)$
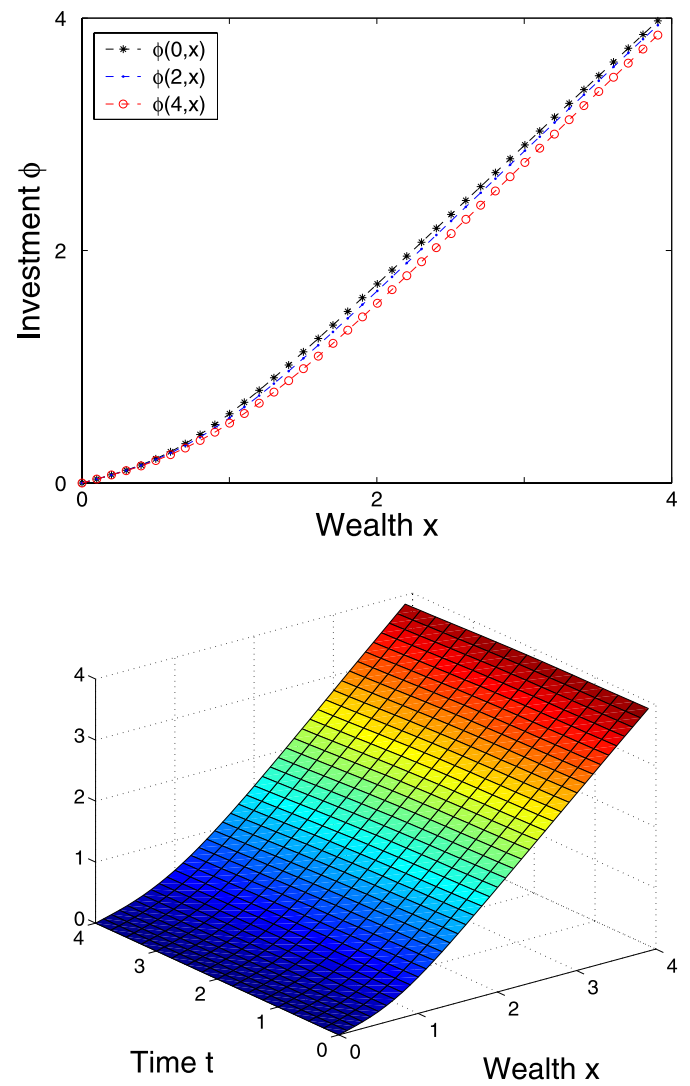

\section{Application to Factorable Models}

In this section we exploit the PDE (13) in problems that show a particular structure, which we call factorable. Suppose $\ell=1$, and that functions $S(x), f(x, u)=$ $f_{0}(x) f_{1}(u)$ and $\sigma(x, u)=\sigma_{0}(x) \sigma_{1}(u)$ are of class $C^{2}$ and independent of time. Assume that the products $f_{0} f_{1}^{\prime}$ and $\sigma_{0} \sigma_{1}^{\prime}$ are different from zero. We also assume that for any constant value $v$ in the control region $U$, the SDE

$$
d X=f_{0}(X) f_{1}(v) d s+\sigma_{0}(X) \sigma_{1}(v) d w(s)
$$

admits a unique strong solution, for any initial condition $(t, x)$.

We impose the following structural conditions on the data.

(i) There exists a constant $\kappa$ such that

$$
f_{0}(x) S^{\prime}(x)=\kappa \sigma_{0}^{2}(x) S^{\prime \prime}(x), \quad \forall x \in \mathbb{R}
$$


(ii) There exists $\lambda$, interior to the control region $U$, such that

$$
\kappa f_{1}^{\prime}(\lambda)=-\sigma_{1}(\lambda) \sigma_{1}^{\prime}(\lambda)
$$

(iii) The following product does not depend on $x$

$$
a:=\left(f_{0}^{\prime}(x)+\frac{1}{\kappa} \frac{f_{0}^{2}(x)}{\sigma_{0}^{2}(x)}\right)\left(f_{1}(\lambda)+\frac{1}{2} \frac{\sigma_{1}^{2}(\lambda)}{\kappa}\right) .
$$

Although these conditions may seem stringent, they are fulfilled in some interesting and common models, such as Merton's problem, as will be shown below. Our claim is the following.

Proposition 6.1 Suppose that for a factorable model, assumptions (i)-(iii) hold. If G is concave with respect to $u$, and $S$ is polynomially bounded, then the optimal control is constant, $\widehat{\phi}(t, x)=\lambda$, with $\lambda$ defined in (i)-(ii). Moreover, the value function is given by

$$
V(t, x)= \begin{cases}e^{-\delta(T-t)}\left(e^{a(T-t)} S(x)+\left(1-e^{a(T-t)}\right)\left(S(\alpha)-\frac{f_{0}(\alpha)}{m(\alpha)} S^{\prime}(\alpha)\right)\right), & \text { if } a \neq 0 ; \\ e^{-\delta(T-t)}\left(S(x)+(T-t) f_{0}(\alpha) S^{\prime}(\alpha)\left(f_{1}(\lambda)+\frac{1}{2} \frac{\sigma_{1}^{2}(\lambda)}{\kappa}\right)\right), & \text { if } a=0,\end{cases}
$$

where a was defined in (ii) above, $\alpha$ is an arbitrary constant, and $m(\alpha)=f_{0}^{\prime}(\alpha)+$ $(1 / \kappa)\left(f_{0}^{2}(\alpha) / \sigma_{0}^{2}(\alpha)\right)$.

Proof With $\kappa$ defined in (ii), we have

$$
F(x, u)=\left(\frac{-f_{1}^{\prime}(u)}{\sigma_{1}(u) \sigma_{1}^{\prime}(u)}\right)\left(\frac{f_{0}(x)}{\sigma_{0}^{2}(x)}\right), \quad \text { hence } F(x, \widehat{\phi})=\frac{1}{\kappa} \frac{f_{0}(x)}{\sigma_{0}^{2}(x)},
$$

which is independent of $t$. On the other hand

$$
\begin{aligned}
& G(t, x, \widehat{\phi}, 1, F)=f_{0}(x)\left(f_{1}(\lambda)+\frac{1}{2} \frac{\sigma_{1}^{2}(\lambda)}{\kappa}\right), \\
& G\left(t, x, \widehat{\phi}, F, F^{2}\right)=\frac{f_{0}^{2}(x)}{\kappa \sigma_{0}^{2}(x)}\left(f_{1}(\lambda)+\frac{1}{2} \frac{\sigma_{1}^{2}(\lambda)}{\kappa}\right) .
\end{aligned}
$$

Thus, the PDE (18) for $F$ is fulfilled because

$$
L^{\widehat{\phi}}(t, x)=\left(\frac{f_{0}^{2}(x)}{\kappa \sigma_{0}^{2}(x)}+f_{0}^{\prime}(x)\right)\left(f_{1}(\lambda)+\frac{1}{2} \frac{\sigma_{1}^{2}(\lambda)}{\kappa}\right)=a
$$

by (iii). Thus, the constant control $\widehat{\phi} \equiv \lambda$ satisfies (18), since $L^{\widehat{\phi}}$ is also constant. Regarding the final condition, it is given by $S^{\prime}(x) F(T, x, \widehat{\phi})=S^{\prime \prime}(x)$, which is simply (i).

To find the value function and show the optimality of $\widehat{\phi}$, we will use Theorem 4.1, hence we find first the adjoint feedback. As has just been shown, $L^{\widehat{\phi}}(t, x)=a$ for 
all $(t, x)$, hence function $F$ evaluated at the optimal control is $F^{\widehat{\phi}}(t, z)=\frac{1}{\kappa} \frac{f_{0}(z)}{\sigma_{0}^{2}(z)}=$ $\frac{S^{\prime \prime}(z)}{S^{\prime}(z)}=\left(\ln S^{\prime}\right)^{\prime}(z)$ for all $(t, z)$, where it has been used (i) in the second equality. In accordance with (20), the adjoint variable is

$$
\gamma(t, x)=S^{\prime}(\alpha) e^{\int_{\alpha}^{x}\left(\ln S^{\prime}\right)^{\prime}(z) d z+a(T-t)}=e^{a(T-t)} S^{\prime}(x) .
$$

The generalized Hamiltonian evaluated at the optimal control is

$$
\mathcal{G}^{\widehat{\phi}}(t, x)=e^{a(T-t)}\left(f_{0}(x) f_{1}(\lambda) S^{\prime}(x)+\frac{1}{2} \sigma_{0}^{2}(x) \sigma_{1}^{2}(\lambda) S^{\prime \prime}(x)\right)
$$

and using this, it is straightforward to find the value function by means of (24), once conditions (i)-(iii) are used. Since $V$ is polynomially bounded, Theorem 4.1 applies, showing that $\widehat{\phi}=\lambda$ is optimal.

This result provides a solution to the HJB equation

$$
\begin{aligned}
& V_{t}(t, x)+\max _{u \in \mathbb{R}}\left\{f_{0}(x) f_{1}(u) V_{x}(t, x)+\frac{1}{2} \sigma_{0}^{2}(x) \sigma_{1}^{2}(u) V_{x x}(t, x)\right\}=\delta V(t, x), \\
& V(T, x)=S_{0}(x),
\end{aligned}
$$

and a (constant) maximizing control, under conditions (i)-(iii). At first sight, it is not apparent what the solution of the HJB equation be; it is even difficult to obtain the explicit form of this non-linear equation, since the maximization cannot be carried out explicitly.

Let us illustrate the use of conditions (i)-(iii) above in some specific models.

\subsection{Logarithm Utility Function}

Consider the problem of maximizing $e^{-\delta(T-t)} \mathrm{E}_{t x}\{\ln X(T)\}$ subject to

$$
d X=b u X \ln X d s+\sigma u X \sqrt{\ln X} d w(s),
$$

with initial condition $X(t)=x>1$, constants $b>0, \sigma>0$, and control region $U=$ $[0, \infty)$. Let us check that (i)-(iii) are fulfilled for suitable constants $\kappa$ and $\lambda$. Here, $f_{0}(x)=x \ln x$ and $\sigma_{0}(x)=x \sqrt{\ln x}$, thus (i) holds if and only if $\kappa=-1$, and then (ii) gives $\lambda=b / \sigma^{2}>0$; finally, it is easy to see that (iii) is satisfied for $a=b^{2} /\left(2 \sigma^{2}\right)$. Thus, the constant control $\widehat{\phi}=b / \sigma^{2}$ is a solution of the PDE and satisfies the final condition. Note that, under $\widehat{\phi}$, the process $Y=\ln X$ has the dynamics

$$
d Y=\frac{b^{2}}{2 \sigma^{2}} Y d s+\frac{b}{\sigma} \sqrt{Y} d w(s),
$$

with $Y(t)=\ln x>0$. This process is positive with probability one and admits a unique solution. Hence, $X>1$ with probability one and the process $X$ is well defined. The generalized Hamiltonian $G$ is concave with respect to $u$ because

$$
G_{u u}=\sigma^{2} x^{2} e^{-a(T-t)} \ln x=-\sigma^{2} e^{-a(T-t)} \ln x<0 .
$$


Since $S(\alpha)-S^{\prime}(\alpha)\left(f_{0}(\alpha) / m(\alpha)\right)=0$, by Proposition 6.1 we have that the value function is $V(t, x)=\exp \left(\left(b^{2} /\left(2 \sigma^{2}\right)-\delta\right)(T-t)\right) \ln x$.

\subsection{Merton's Problem for a Large Investor}

Consider again the problem of Merton introduced in Example 5.2, now considering $u(s)$ not as the total wealth invested in the risky asset, but the proportion of wealth invested. Both formulations are equivalent. Consider also the case where the coefficients are constant. The model is factorable, with $f_{0}(x)=\sigma_{0}(x)=x$, $f_{1}(u)=r+b u$ and $\sigma_{1}(u)=\sigma u$. Assumption (i) holds with $\kappa=-\rho^{-1}$ for HARA utilities $S(x)=\frac{K}{1-\rho} x^{1-\rho}$, where $K>0, \rho>0, \rho \neq 1$, or $S(x)=K \ln x$ (this case could be embedded in the above with $\rho=1$ ), which were already considered in Sect. 5.2. Using (ii) we get the constant control: $\widehat{\phi}=\lambda=\rho^{-1}\left(b / \sigma^{2}\right)$ - that obviously agrees with our findings in Sect. 5.2 - and (iii) gives

$$
a=(1-\rho)\left(r+b \lambda-(1 / 2) \rho \sigma^{2} \lambda^{2}\right)=(1-\rho)\left(r+(1 / 2) \rho^{-1}\left(b^{2} / \sigma^{2}\right)\right) .
$$

According to Proposition 6.1, the value function is

$$
V(t, x)=e^{(a-\delta)(T-t)} S(x)=e^{(a-\delta)(T-t)} K \frac{x^{1-\rho}}{1-\rho} .
$$

Note that $G$ is concave with respect to $u$ because $G_{u u}=\sigma^{2} x^{2} V_{x x}<0$.

Nothing new in the above, of course, but consider the following variation of the problem, which could be applicable in financial economics. Suppose, as in [17], that the investor is large, in the sense that his/her investment decisions influence the evolution of the market price of the asset. We are thinking of large financial institutions whose performance (benefits or losses) affect the global financial market. Well known examples of this possibility were the crashes due to the "Hedge Fund Crisis" of 1998 and some others, more recent cases. Nevertheless, our model is only theoretical, with a view to illustrating our results.

Suppose that the price of the risky asset is given by

$$
d P^{1}(t)=(b(u)+r) P^{1}(t) d t+\sigma(u) P^{1}(t) d w(t), \quad P^{1}(0) \text { known. }
$$

We observe that the investment decisions of the investor influence the price dynamics through functions $b$ and $\sigma$, which we consider to be of class $C^{2}$ and positive. The riskless return $r$ is supposed to be constant. Then, the wealth evolves as

$$
d X(s)=X(s)(r+u(s) b(u(s))) d t+X(s) u(s) \sigma(u(s)) d w(s), \quad t \leq s \leq T .
$$

Thus, we identify a factorable dynamics, with

$$
f_{0}(x)=\sigma_{0}(x)=x, \quad f_{1}(u)=r+u b(u), \quad \sigma_{1}(u)=u \sigma(u) .
$$

Consider again a HARA utility function. We have already tested that condition (i) holds with $\kappa=-\rho^{-1}$. For (ii), let us be more specific. Choose $b(u)=b_{1} \exp \left(-b_{2} u\right)$ and $\sigma(u)=\sigma$ with $b_{1}$, and $\sigma$ positive constants, and let $b_{2}$ be non-negative, satisfying 
$-\rho^{-1} b_{1} \exp \left(-b_{2}\right)\left(1-b_{2}\right)+\sigma^{2}>0$. Note that $b_{2}=0$ gives the Merton problem for a small investor, where the above inequality means that the optimal proportion of wealth invested in the risky asset is not bigger than one, which is a desirable featureit says that no borrowing is allowed-. Wealth dynamics becomes

$$
d X(s)=X(s)\left(r+b_{1} u(s) e^{-b_{2} u(s)}\right) d t+X(s) \sigma u(s) d w(s), \quad t \leq s \leq T .
$$

Condition (ii) gives the equation

$$
-\rho^{-1} b_{1} \exp \left(-b_{2} \lambda\right)\left(1-b_{2} \lambda\right)+\sigma^{2} \lambda=0
$$

for the determination of $\lambda$. The function $h(u)=-\rho^{-1} b_{1} \exp \left(-b_{2} u\right)\left(1-b_{2} u\right)+\sigma^{2} u$ is continuous and by assumption, $h(1)=-\rho^{-1} b_{1} \exp \left(-b_{2}\right)\left(1-b_{2}\right)+\sigma^{2}>0$. Since $h(0)=-\rho^{-1} b_{1}<0$ and $h$ is strictly increasing in $[0,1],(42)$ admits a unique solution, $\widehat{\phi}=\lambda \in(0,1)$, which is the constant optimal control. Finally, (iii) is satisfied since the left-hand side factor defining $a$ is independent of $x$. Thus, large investors with HARA utility may behave as in the typical Merton's problem, where the investment decisions do not affect the price; that is, the optimal investment rule for large investors is also proportional to total wealth as it is for small investors.

The value function of the problem can also be found

$$
V(t, x)=\exp ((a-\delta)(T-t)) K(1-\rho)^{-1} x^{1-\rho},
$$

where $a=(1-\rho)\left(r+b_{1} \lambda \exp \left(-b_{2} \lambda\right)-(1 / 2) \rho \sigma^{2} \lambda^{2}\right)$. Finally, the generalized Hamiltonian $G$ is concave with respect to $u$ in the relevant range of values $[0,1]$, since the value function is strictly increasing and strictly concave. This can be verified by testing that, selecting $b_{2}<2$, the second order derivative

$$
G_{u u}=b_{1} b_{2} e^{-b_{2} u}\left(b_{2} u-2\right) x V_{x}+\sigma^{2} x^{2} V_{x x}<0, \quad \text { for all } u \in[0,1] .
$$

Of course, conditions (i)-(iii) apply to much more general pairs of functions $b, \sigma$. From the economic point of view, our selection implies that, by buying the risky asset, the investor diminishes the mean market price of the risky stock, having no effect on its volatility. For general $b$ and $\sigma$, condition (ii) is $\kappa(\lambda b(\lambda))^{\prime}+(\lambda \sigma(\lambda))(\lambda \sigma(\lambda))^{\prime}=0$ for some $\lambda \in[0,1]$, which should be tested for the specific model at hand. Obviously, the concavity of the generalized Hamiltonian can also be studied as in the parametric example considered.

We have selected the particular pair $b, \sigma$ above as an illustration of the result, and to show the difficulties associated in solving the problem by means of the HJB equation. Whilst with our methods the problem has been solved fairly well, finding the solution with the HJB equation is difficult, as the maximization condition

$$
b_{1} x e^{-b_{2} u}\left(1-b_{2} u\right) V_{x}+x^{2} \sigma^{2} u V_{x x}=0
$$

cannot be solved explicitly to express $u$ in terms of $V_{x}, V_{x x}$. Thus, the PDE (8) for the adjoint feedback $\gamma$ cannot be explicitly given, since

$$
\frac{\gamma_{x}}{\gamma}=F=-\frac{b_{1} e^{-b_{2} u}\left(1-b_{2} u\right)}{\sigma^{2} x u}
$$

cannot be solved for $u=\bar{u}\left(x, \gamma, \gamma_{x}\right)$. 


\section{Conclusions}

This paper proposes a systematic method to find a PDE for the optimal control to study one-dimensional stochastic models of Mayer type. These models are usual in financial economics, as in the mean variance portfolio problem or the Merton problem without consumption. The PDE for the optimal control is a Euler companion to the usual HJB equation. Whereas this equation is fully non-linear, the former is of quasilinear type. This fact allows us to show the existence of the optimal control in the Merton problem with time varying (but deterministic) coefficients, even for utility functions with an unbounded absolute risk tolerance index (but with at most linear growth). We also provide sufficient conditions in terms of the PDE found, similar to the verification theorems in [7]. The connection between the optimal control and the value function is explicitly found through the adjoint feedback $\gamma$. Finally, the theory is applied to a family of problems that are factorable in the state and the control variable, showing how the new PDE helps in determining the (a priori hidden) solution in the form of a constant control. This class contains the classical Merton's problem as well as the problem which considers a large investor whose decision can influence the price of the stock. Surprisingly enough, it is shown that the optimal investment rule of this problem with non-linear dynamics is proportional to the wealth level, as in the traditional model, when the investor shows HARA preferences.

\section{Appendix}

We show here how the PDE (17) is obtained.

Consider the PDE (13),

$$
\begin{gathered}
\frac{\partial}{\partial t} F(t, x, \phi)+\frac{\partial}{\partial x} G\left(t, x, \phi, F(t, x, \phi), F^{2}(t, x, \phi)\right) \\
+\frac{\partial^{2}}{\partial x^{2}} G(t, x, \phi, 1, F(t, x, \phi))=0 .
\end{gathered}
$$

The first summand is:

$$
\frac{\partial}{\partial t} F(t, x, \phi)=F_{t}+F_{u} \phi_{t} .
$$

Recalling that

$$
\begin{aligned}
G\left(t, x, \phi, F(t, x, \phi), F^{2}(t, x, \phi)\right)= & f(t, x, \phi) F(t, x, \phi) \\
& +\frac{1}{2} \sigma(t, x, u) \sigma^{\top}(t, x, u) F^{2}(t, x, \phi),
\end{aligned}
$$

then second summand is

$$
\begin{aligned}
& \frac{\partial}{\partial x} G\left(t, x, \phi, F(t, x, \phi), F^{2}(t, x, \phi)\right) \\
& \quad=f_{x} F+f F_{x}+\sigma_{x} \sigma^{\top} F^{2}+\sigma \sigma^{\top} F F_{x}
\end{aligned}
$$




$$
\begin{aligned}
&+(\overbrace{\left(f_{u}+\sigma_{u} \sigma^{\top} F\right.}^{=0(\text { by }(7))} F+f F_{u}+\sigma \sigma^{\top} F F_{u}) \\
& \phi_{x} \\
&= f_{x} F+f F_{x}+\sigma_{x} \sigma^{\top} F^{2}+\sigma \sigma^{\top} F F_{x}+\left(f+\sigma \sigma^{\top} F\right) F_{u} \phi_{x} .
\end{aligned}
$$

In the same way, taking into account that

$$
G(t, x, \phi, 1, F(t, x, \phi))=f(t, x, \phi)+\frac{1}{2} \sigma(t, x, u) \sigma^{\top}(t, x, u) F(t, x, \phi),
$$

the total derivative with respect to $x$ is

$$
\begin{aligned}
& \frac{\partial}{\partial x} G(t, x, \phi, 1, F(t, x, \phi)) \\
& \quad=f_{x}+\sigma_{x} \sigma^{\top} F+\frac{1}{2} \sigma \sigma^{\top} F_{x}+(\overbrace{f_{u}+\sigma_{u} \sigma^{\top} F}^{=0}+\frac{1}{2} \sigma \sigma^{\top} F_{u}) \phi_{x} \\
& =f_{x}+\sigma_{x} \sigma^{\top} F+\frac{1}{2} \sigma \sigma^{\top} F_{x}+\frac{1}{2} \sigma \sigma^{\top} F_{u} \phi_{x} .
\end{aligned}
$$

Deriving again in the last expression we get

$$
\begin{aligned}
\frac{\partial^{2}}{\partial x^{2}} G(t, x, \phi, 1, F(t, x, \phi)) \\
=\frac{\partial}{\partial x}\left(f_{x}+\sigma_{x} \sigma^{\top} F+\frac{1}{2} \sigma \sigma^{\top} F_{x}+\frac{1}{2} \sigma \sigma^{\top} F_{u} \phi_{x}\right) \\
=f_{x x}+\sigma_{x x} \sigma^{\top} F+\sigma_{x} \sigma_{x}^{\top} F+2 \sigma_{x} \sigma^{\top} F_{x}+\frac{1}{2} \sigma \sigma^{\top} F_{x x} \\
\quad+\left(f_{x u}+\sigma_{x u} \sigma^{\top} F+\sigma_{x} \sigma_{u}^{\top} F+2 \sigma_{x} \sigma^{\top} F_{u}+\sigma_{u} \sigma^{\top} F_{x}+\sigma \sigma^{\top} F_{x u}\right) \phi_{x} \\
\quad+\left(\sigma_{u} \sigma^{\top} F_{u}+\frac{1}{2} \sigma \sigma^{\top} F_{u u}\right) \phi_{x}^{2}+\frac{1}{2} \sigma \sigma^{\top} F_{u} \phi_{x x} .
\end{aligned}
$$

Putting together all the above expressions we arrive to

$$
\begin{aligned}
F_{t} & +F_{u} \phi_{t}+f_{x x}+\left(f_{x}+\sigma_{x x} \sigma^{\top}+\sigma_{x} \sigma_{x}^{\top}\right) F+\left(f+\sigma \sigma^{\top} F+2 \sigma_{x} \sigma^{\top}\right) F_{x} \\
& +\sigma_{x} \sigma^{\top} F^{2}+\frac{1}{2} \sigma \sigma^{\top} F_{x x} \\
& +\left(f_{x u}+\left(f+\sigma \sigma^{\top} F+2 \sigma_{x} \sigma^{\top}\right) F_{u}+\left(\sigma_{x u} \sigma^{\top}+\sigma_{x} \sigma_{u}^{\top}\right) F+\sigma_{u} \sigma^{\top} F_{x}\right. \\
& \left.+\sigma \sigma^{\top} F_{x u}\right) \phi_{x}+\left(\sigma_{u} \sigma^{\top} F_{u}+\frac{1}{2} \sigma \sigma^{\top} F_{u u}\right) \phi_{x}^{2}+\frac{1}{2} \sigma \sigma^{\top} F_{u} \phi_{x x}=0 .
\end{aligned}
$$

Now we divide the PDE (43) by $F_{u} \neq 0$ to get (17). 


\section{References}

1 Bourdache-Siguerdidjane, H , Fliess, M : Optimal feedback control of nonlinear systems Automatica 23, 365-372 (1987)

2 Rincón-Zapatero, J P, Martínez, J , Martín-Herrán, G : New method to characterize subgame perfect Nash equilibria in differential games J Optim Theory Appl 96, 377-395 (1998)

3 Rincón-Zapatero, J P: Characterization of Markovian equilibria in a class of differential games J Econ Dyn Control 28, 1243-1266 (2005)

4 Josa-Fombellida, R, Rincón-Zapatero, J P: New approach to stochastic optimal control J Optim Theory Appl 135, 163-177 (2007)

5 Huang, C, Zariphopoulou, T: Turnpike behavior of long-term investments Finance Stoch 3, 15-34 (1999)

6 Schied, A, Schöneborn, T : Risk aversion and dynamics of optimal liquidation strategies in illiquid markets Finance Stoch 13, 181-204 (2009)

7 Fleming, W H , Soner, H M : Controlled Markov Processes and Viscosity Solutions Springer, New York (2006)

8 Yong, J, Zhou, X Y : Stochastic Controls Hamiltonian Systems and HJB Equations Springer, New York (1999)

9 Danskin, J M : The Theory of Max Min and Its Applications to Weapon Allocation Springer, Berlin (1967)

10 Bismut, J M : Conjugate convex functions in optimal stochastic control J Math Anal Appl 44, 384-404 (1973)

11 Rogers, L C G : Duality in constrained optimal investment and consumption problems: a synthesis In: Paris-Princeton Lectures on Mathematical Finance 2002 Springer Lecture Notes in Mathematics, vol 1814, pp 95-131 Springer, Berlin (2003)

12 Karatzas, I, Lehoczy, J P, Sheti, S, Shreve, S E : Explicit solutions of a general consumption/investment problem Math Oper Res 11, 261-294 (1986)

13 Karatzas, I : Optimization problems in continuous trading SIAM J Control Optim 27, 1221-1259 (1989)

14 Merton, R C : Optimum consumption and portfolio rules in a continuous time model J Econ Theory 3, 373-413 (1971)

15 Ladyzhenskaya, O A, Solonnikov, VA, Ural'ceva, N N : Linear and Quasi-linear Equations of Parabolic Type Translations of Mathematical Monographs, vol 23 American Mathematical Society, Providence (1968)

16 Constantin, A, Escher, J : Global solutions for quasilinear parabolic problems J Evol Equ 2, 97-111 (2002)

17 Cuoco, D, Cvitanić, J : Optimal consumption choices for a 'large' investor J Econ Dyn Control 22, 401-436 (2000) 